\title{
Guiding the Interactive Exploration of Metabolic Pathway Interconnections
}

\author{
Ilir Jusufi, Christian Klukas, Andreas Kerren, Member, IEEE, and Falk Schreiber
}

\begin{abstract}
Approaches to investigate biological processes have been of strong interest in the last years and are in the focus of several research areas, especially Systems Biology. Biochemical networks are very important for such a comprehensive understanding of living beings. Drawings of these networks are often visually overloaded and do not scale. A common solution to deal with this complexity is to divide the complete network, e.g., the metabolism, into a large set of single pathways that are hierarchically structured. If those pathways are visualized, this strategy generates additional navigation and exploration problems as the user looses the context within the complete network. In this paper, we present a general solution of this problem of visualizing interconnected pathways and discuss it in context of biochemical networks. Our new visualization approach supports the analyst to get an overview to related pathways if he/she is working within a particular pathway of interest. By using glyphs, brushing, and topological information of the related pathways, our interactive visualization tool is able to intuitively guide the exploration and navigation process, and thus the analysis processes too. To deal with real data and current networks, our tool has been implemented as plugin for the VANTED system.
\end{abstract}

Index Terms-Network visualization, biochemical networks, navigation.

\section{INTRODUCTION}

The visualization of large and complex networks is still one of the most challenging tasks in graph drawing and information visualization [15]. Even if considerable work on graph visualization has been done in the literature and powerful tools exists, some fundamental problems are not sufficiently solved. One example of such a problem is the embedding of additional information that is attached to the network nodes and edges. Another example is the well-known scalability problem that comes up if we try to visualize huge networks. Drawings of these networks are often visually overloaded and do not scale. To focus on the last mentioned problem, one traditional solution is a hierarchical structuring of the entire network if possible, i.e., the complete network is divided into many parts. This is also a common procedure in biochemical network drawings.

Approaches to investigate biological processes have been of strong interest in the last years. Biochemical networks are crucial for a comprehensive understanding of living beings and dividing a metabolic network into a large set of single pathways, for example, that are hierarchically structured is currently the state-of-the-art strategy [10], see also Section 2. If these pathways are visualized, then additional navigation and exploration problems occur, because the user looses the overview on the entire network. Similar problems can be identified in other domains, such as in the visualization of communication networks, social networks, etc.

In this paper, we present a general approach of navigating between interconnected subgraphs and discuss it in context of biochemical networks, i.e., in a set of hierarchically structured pathways (groups and

- Ilir Jusufi and Andreas Kerren are with Linnaeus University, School of Computer Science, Physics and Mathematics (DFM), Vejdes Plats 7, 351 95 Växjö, Sweden, E-mail: \{ilir.jusufi, andreas.kerren\}@lnu.se.

- Christian Klukas is with Leibniz Institute of Plant Genetics and Crop Plant Research (IPK) Gatersleben, Corrensstr. 3, 06466 Gatersleben, Germany,E-mail: klukas@ipk-gatersleben.de.

- Falk Schreiber is with Institute of Computer Science, Martin Luther University Halle-Wittenberg, Von-Seckendorff-Platz 1, 06120 Halle, Germany, and Leibniz Institute of Plant Genetics and Crop Plant Research (IPK) Gatersleben, Corrensstr. 3, 06466 Gatersleben, Germany, E-mail: schreibe@ipk-gatersleben.de.

Manuscript received 31 March 2009; accepted 27 July 2009; posted online 11 October 2009; mailed on 5 October 2009.

For information on obtaining reprints of this article, please send email to: tvcg@computer.org . sub-groups of pathways). Our new visualization approach initially supports the researcher to get an overview on pathways, which are connected to the currently considered pathway via its nodes and edges. Based on this context information, our interactive visualization tool is able to intuitively guide the exploration and navigation process by using glyphs, brushing, and topological information of the involved pathways. In this way, it can help the analyst to perform the analysis processes more efficiently. To deal with real data, current networks and concrete problems, we decided to implement our approach as a plugin for the VANTED system. This system was originally developed for the visualization and analysis of experimental data in biochemical networks.

Our paper is organized as follows: Background information and related work, concerning the biological as well as visualization aspects are presented in Section 2. The underlying VANTED system is briefly illustrated in Section 3, while details of our novel navigation and exploration approach are given in Section 4. Here, we also provide an overview of limitations and implementation issues. A use-case scenario is discussed in the next section followed by a conclusion and ideas for future improvements.

\section{Background AND Related Work}

Biological networks such as gene regulatory networks, protein interaction networks and metabolic networks play an important role in the investigation of biological processes. They are crucial for a comprehensive understanding of living beings. However, due to the steady progress of knowledge, which result in increasingly large and complex networks, these networks are increasingly difficult to interpret and visualize. An example is network information managed in the KEGG database [10], which contains hierarchically structured pathways with in total more than 10,000 nodes representing genes, proteins/enzymes, and metabolites.

To deal with such large networks several methods and tools have been developed. Simple approaches try to visualize the complete network on the screen and use zooming and panning for navigation (examples are common graph drawing or network analysis tools). As such large networks are not easily understandable, they are often separated in overlapping pathways. Commonly these pathways are structured hierarchically, for example, in metabolism several pathways representing the synthesis of amino acids are grouped into a super-pathway Amino Acid Metabolism. Dividing networks into pathways help reducing the overall complexity, but has the disadvantage that a user looses the context. An example is the Web-interface of the KEGG database [10], which allows to navigate from one pathway image by 
clicking onto a link to the next, connected pathway image. However, this approach means that connections to elements in other pathways are hard to follow, and that many of the network elements are multiplied several times in different pathways. Other approaches, such as KGML-ED [13], try to improve the navigation between pathways by providing an overview (top nodes of the hierarchy) and functions to zoom into such top nodes, or by extending the pathway by connected pathways within the same frame. However, the main problem remains, which is that either too much information (e.g., many or all pathways at once) is shown, and therefore the picture is not readable anymore, or that the connection to other pathways is lost or difficult to follow. MetaViz presented by Bourqui et al. [4] follows a similar approach. It simultaneously visualizes the global network topology as well as the metabolic pathways without multiplying network nodes. Pathways are layouted by using different graph drawing algorithms. In addition, a user-defined set of focus pathways can be drawn entirely and independently due to specific domain requirements. The interconnection of pathways is displayed by using an orthogonal graph drawing algorithm. This strategy leads to long orthogonal edges, which are often hard to follow. Moreover, the connection overview is lost in case the user needs to zoom into specific pathways. In contrast, our approach provides an overview of the interconnections between a single focus pathway and connected pathways, instead of providing a general overview with additional edges. To the best of our knowledge, biologists usually have no problem with node redundancies. They are more interested to recognize "their" pathways during the exploration process. A good overview on open problems and challenges in biological network visualization is provided by Albrecht et al. [1,2].

Current approaches are mostly based on a close interdisciplinary work between visualization or graph drawing researchers and domain experts, such as in biochemistry. Saraiya et al. [23] discussed requirements of biochemical network visualization collected from interviews with domain experts. The authors observed five crucial needs that are important for biologists working on pathway analysis, but still not completely realized in existing visualization tools. Our approach presented in this paper realizes a 2D solution for the need to overview "multiple pathways simultaneously with interconnections between them" [16]. Specifically, "incoming and outgoing visual links could enable users to view how other pathways can potentially affect or be affected by the focus pathway at each node. In a densely populated pathway, it is important to be able to analyze connectivity between components" [23]. A related project is the Caleydo framework [27] that extends the KEGG pathways drawings into $2.5 \mathrm{D}$, similar to the work of Kerren [11] or Brandes et al. [5], combined with brushing, highlighting, focus\&context, and detail on demand. In this way, it supports the interactive exploration and navigation between several interconnected components by using the 3rd dimension. Drawbacks of this approach are the restricted number of connected pathways that can be displayed because of the bucket-like visualization setup and the unavoidable clutter/overlaps in 3D if a node-link metaphor is used.

Recently, Rohrschneider et al. [22] presented a grid-based visualization approach for metabolic networks supported by a focus\&context view. This view is based on a Table Lens method [19], which provides multiple foci and together with the grid-layout the preservation of the user's mental map [17]. But even by using these rich navigation and interaction possibilities the cognitive overload is still high and the approach follows a classical top-down navigation method. In case the analyst starts from a single node, he/she has to follow the links to other pathways successively. Here in this paper, we present a technique in order to improve this situation. Because of space restrictions, we do not give a comprehensive overview of other related tools and approaches and refer to the papers [2,22] instead. Both provide a comprehensive list of related work, not only focused on pathway interconnections.

As already mentioned in the introduction, our interactive exploration and navigation approach is based on the VANTED system, which is briefly presented in the following section.

\section{VANTED}

VANTED $[9,14]$ is a flexible system that aims in supporting researchers to visualize and analyze data from experiments in context of biological networks or pathways. The drawing algorithms of VANTED are based on the extensible graph library and editor Gravisto [3]. VANTED can be easily extended by plugins, and several plugins have been developed, e.g., to compute and visualize fluxes in metabolism or to connect databases to VANTED.

In this paper, we use the MetaCrop database [8] that summarizes diverse information about metabolic pathways in crop plants and allows automatic export of information for the creation of detailed metabolic models. MetaCrop is based on the freely available Meta-All system [32] and can be directly accessed by VANTED. In MetaCrop, metabolites are depicted as circular nodes; proteins (enzymes or transporters) are shown as rectangular nodes. In the following screenshot examples, a background coloring is used to visually represent different cell compartments (cf. also Figure 4). This information can optionally be used to restrict links to substances represented in multiple pathways to only those cases, where the substance name and the particular compartment information is matching.

\section{Novel Approaches to Guide the Exploration Pro- CESS}

Our visualization tool is currently focused on MetaCrop pathways, which are placed in six groups (Amino Acid Metabolism, Carbohydrate Metabolism, Cofactor Metabolism, Energy Metabolism, Lipid Metabolism, and Nucleotide Metabolism) consisting of 38 pathways in total. Thus, the MetaCrop hierarchy has two levels only. The relatively small number of pathways and their more or less distinct graph topology makes these graphs easy to identify by professionals (depending on the graph layout), which is an important aspect for our approach discussed in the section.

The original support of VANTED to navigate between interconnected hierarchical pathways is shown in Figure 1. On the right hand side, a pathway hierarchy is displayed in which the oxidative phosphorylation pathway is selected and shown on the left. This pathway is a child of the energy metabolism super-pathway and relatively small. Within the pathway drawing, one node fumarate is in the focus of the user. By mouse-click it is possible to get shown all links to other pathway nodes connected to fumarate. In this example, two rounded rectangles are connected with this node using dashed gray lines, whereas each rectangle represents another pathway. If the user is interested to follow, for example, the path to the TCA cycle (part of the carbohydrate metabolism), then he/she has to click on the corresponding rounded rectangle to open a new window with the target pathway displayed. All subsequent steps occur analogously.

It is easy to understand that this navigation strategy should be improved, because of the unavoidable clutter if the pathways are large and many nodes are selected. Furthermore, it is not really possible to get an overview about all the pathways linked to the currently selected node(s) and about the overall distribution of all outgoing links.

\subsection{Navigation Glyphs}

In a first step to overcome these problems, we developed small navigation glyphs, which are embedded within the network nodes. They show the pathway link situation outgoing from a specific node. Figure 2 shows one instance of a navigation glyph. The motivation behind these rose-shaped diagrams is straightforward as it follows the idea of Nightingale's rose diagram [18] and extend it by reflecting the pathway hierarchy. The color coding of the "glyph petals" represents the pathway group (level 1 in the hierarchy), while their size/length shows if there is a link (to the same node in another pathway) or not. In more detail, our sample glyph in Figure 2 is attached to one specific node in a pathway, and this node is connected to five other pathways displayed in five different colors. The highlighted sector gives an impression about the strength of the connectivity to a specific pathway group (Amino Acid Metabolism in this case). It is divided into 12 petals in sum, representing all pathways belonging to this group, and 6 longer/extended 


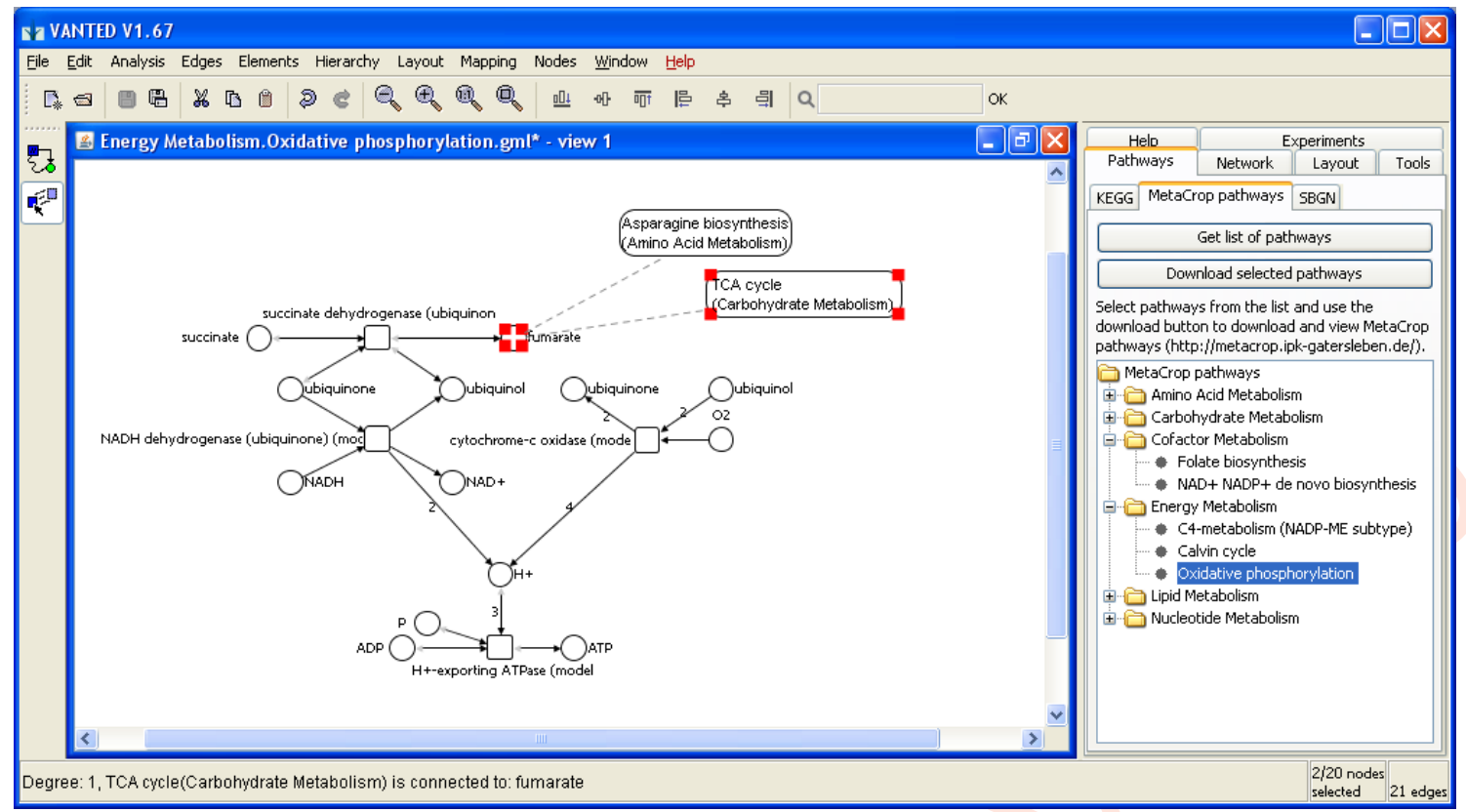

Fig. 1. Original navigation approach between pathways provided by VANTED. Background coloring to show different cell compartments was switched off to facilitate the identification of the different graphical elements by the reader.

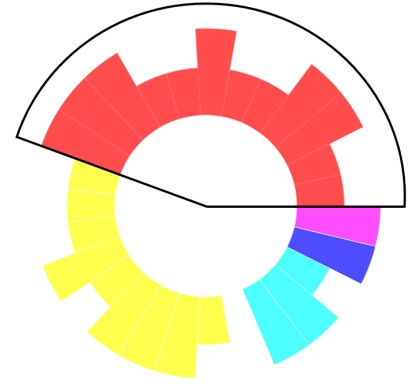

Fig. 2. Navigation Glyph. For the color coding of the pathway group, we use a subset of the standard colors for nominal data types suggested by C. Ware [31, p. 126]. The highlighted sector represents all connected pathways belonging to one super-pathway (or pathway group), e.g., Amino Acid Metabolism.

petals. This means that there are links to 6 sub-pathways of the current group in the second level of the hierarchy. It is possible that such glyphs have gaps in the sense of missing petals as shown in the lower part of the example. Such a fact occurs if the underlying VANTED system detects a pathway group that is completely unconnected to the currently considered node.

Our navigation glyphs provide a good and simple overview about the relationships to connected pathway groups outgoing from a single node, which is a clear improvement compared to the original approach of VANTED. The navigation is then performed using a right click on a node, which will display a context menu with a list of links to all connected pathways, see Figure 3. A certain pathway window is activated after a click on a specific link. In this way, the navigation is comparable to the old approach explained at the beginning of Section 4, and it has also similar drawbacks, such as loosing a direct link to the previous pathway window: in order to return to the previously shown pathway, we need to minimize the newly opened window and find the first one or to directly access the node that has a link to it. Moreover, we have no clear insight into all links of the current pathway, because of the missing interactive mapping of the petals to the connected pathways.

\subsection{Extended Navigation Approach}

Our main idea is to overcome the drawbacks of the navigation glyphs presented in the previous subsection by

1. providing an overview of all connected pathways of the current focus pathway,

2. showing statistical information about the focus pathway's link distribution, and

3. facilitating the navigation from nodes in the focus pathway to different target pathways.

For this, we introduce new visualization and brushing techniques coupled with seamless interaction of various parts of the system. That will make the navigation of different pathways easier and yields a more intuitive exploration experience. We also investigated the need to provide more insight into the links from a particular pathway by introducing a possibility to compare the number of total references to all connected pathways.

\subsubsection{Navigation Window}

The approach consists of laying out minimized images of pathway drawings, called graph icons, around the main view that contains the currently considered focus pathway, see Figure 4. Both together form the navigation window of our VANTED plugin. The graph icons are used to navigate to a specific pathway by mouse clicks, which would load the target pathway into the navigation window. Each graph icon has a background color that is brushed accordingly to the color coding of the navigation glyphs within the pathway nodes, thus making it easy to identify the different pathway groups of the MetaCrop data set. The user is able to recognize the pathways connected to the nodes of the focus pathway based on their graph topology, additionally supported by the aforementioned color coding. As written before, this recognition of the target pathways is of particular importance as the user can directly identify well-known pathways with a catchy and unique layout, such as the TCA cycle. For precise information about the pathways, the user can use tooltips showing the name of the pathway and of the pathway group. They appear based on a mouse-over event of a specific graph icon. 


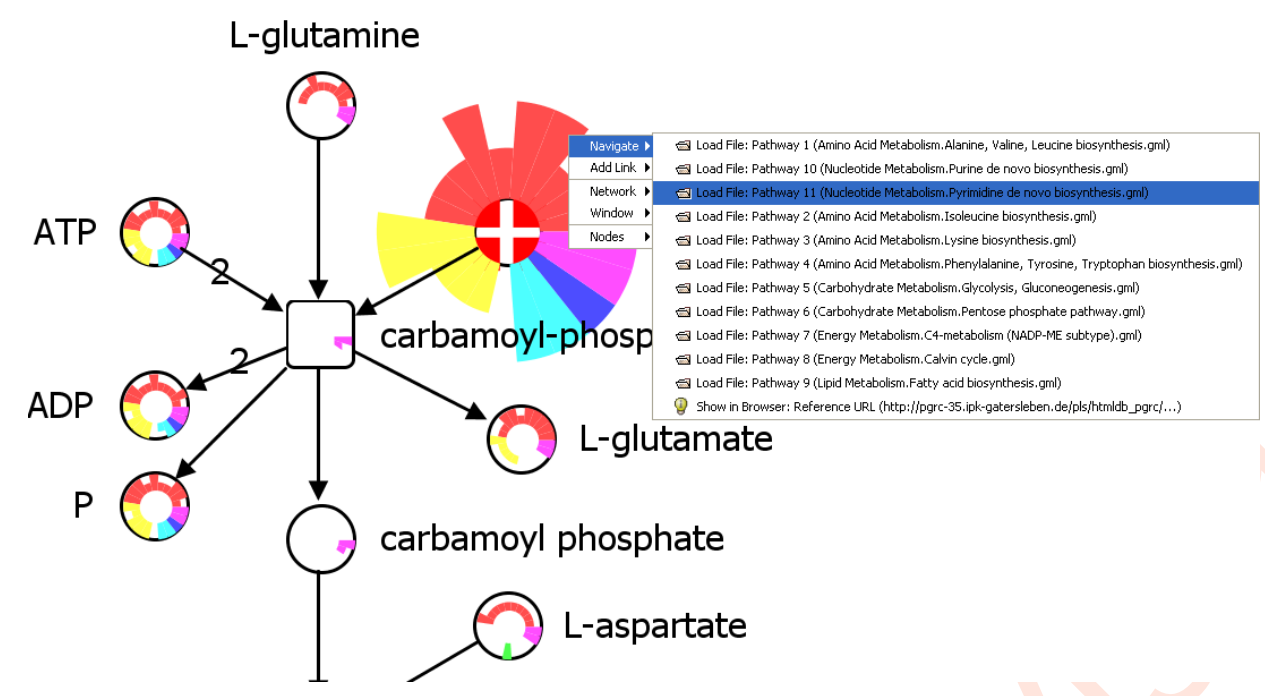

Fig. 3. Embedding of our navigation glyphs into some nodes of a (focus) pathway. The glyph of the currently selected node is highlighted and magnified by the system. With a right mouse-click the user is able to open a context menu with a link list from which he/she can jump to connected pathways. The target pathway is then displayed in a new window.

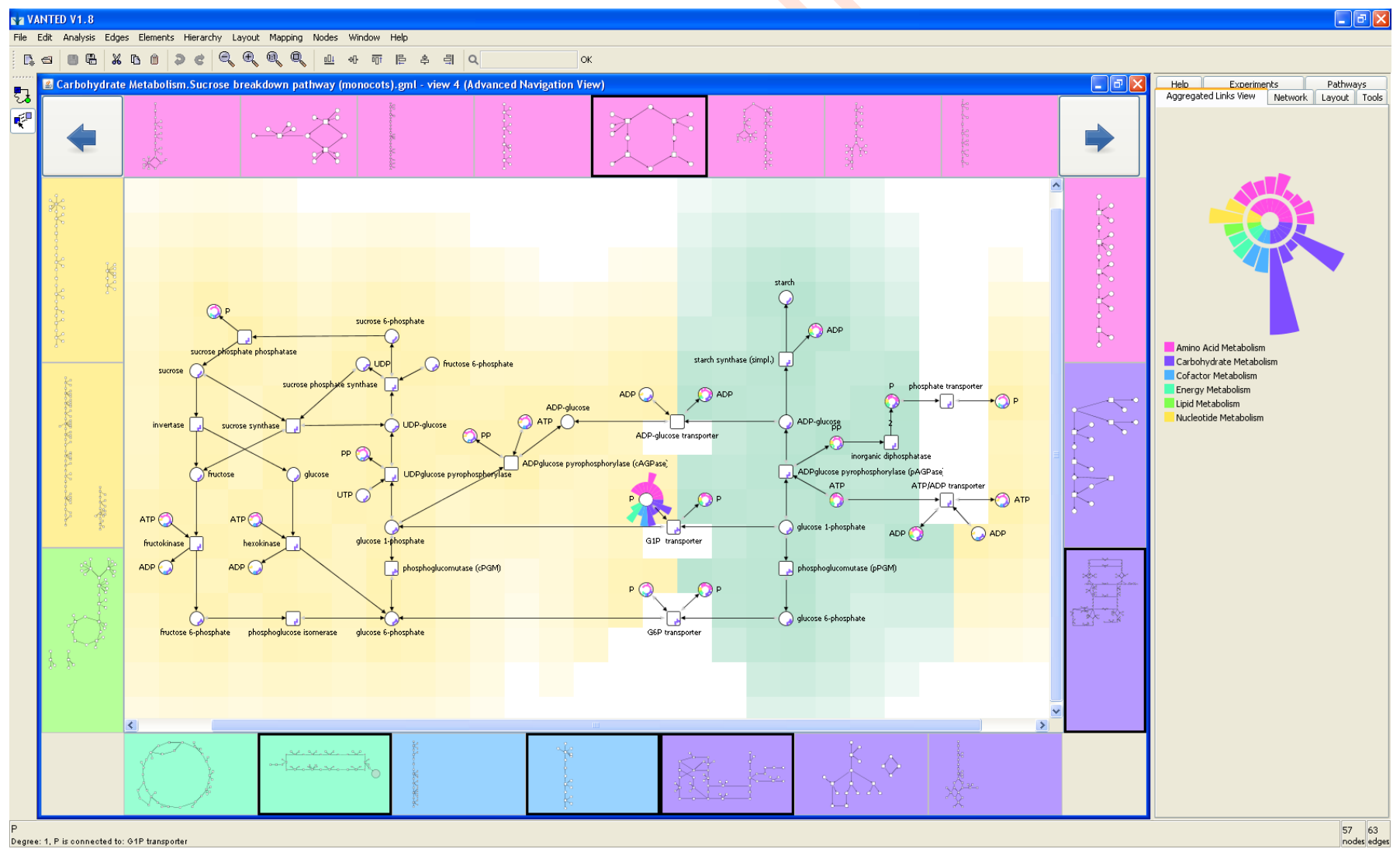

Fig. 4. Overview of the new VANTED plugin with the navigation window on the left and the aggregated links view on the right. The background coloring of the shown focus pathway represents different cell compartments. One node is selected and its navigation glyph was magnified by the system. The glyph petals are directed to the surrounding graph icons. Links to other pathways are double-coded by the longer petals as well as by the highlighted graph icons. The Link Rose Diagram in the aggregated links view (on the right) is explained in Section 4.2.2. 
The position of the graph icons roughly corresponds to the position of the petals within the small navigation glyphs, which in turn provides additional insight to this existing feature. We chose this layout design since our users already used the navigation glyph metaphor, i.e., our solution complements the old one aimed at specific pathway nodes as discussed in Subsection 4.1, while making it more intuitive to use. We consider this to be a better alternative compared to solutions such as making a simple link list that in turn might require scrolling efforts to fit all the links and does not provide a mapping of the pathways with our navigation glyphs. As we will explain later in this paper, we have also implemented an additional visualization view (the aggregated links view) that uses the same layout and navigation concept and provides an integrated tool for link aggregation to facilitate the guided interactive exploration.

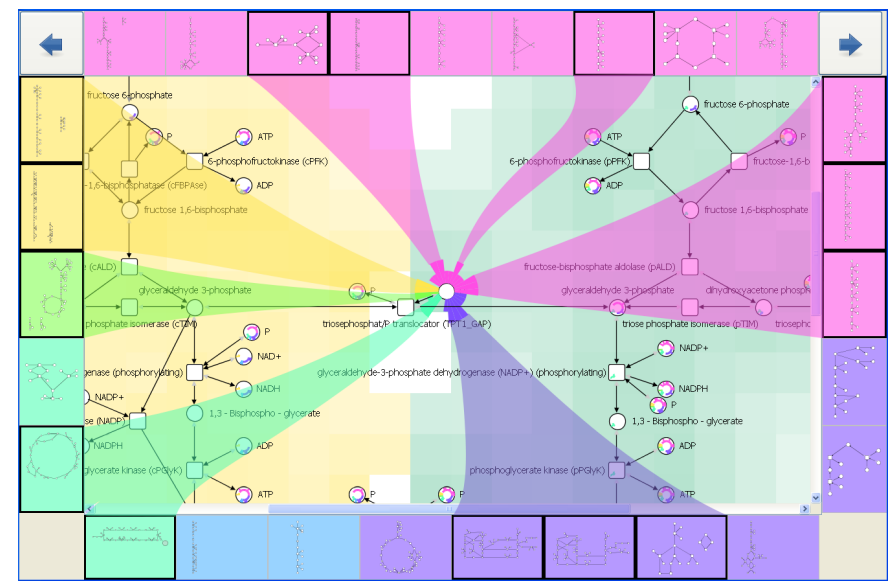

Fig. 5. A mouse-over action on a specific node highlights the correlated graph icons (black framed) around the focus pathway. Note that the halftransparent shapes are hand made and are not part of our plugin; they are just drawn to demonstrate the ordering and layout of the approach.

The previous approach with exclusive navigation glyphs allowed navigation to different pathways by activating separate views through a context menu for each linked pathway as it was explained earlier. By the graph icons, it is now possible to navigate through all related pathways using one window, while providing a good overview of all pathway links. Users can easily access all pathways linked from an interesting node through a mouse-over, which will highlight the corresponding graph icon, cf. Figure 5. This feature allows users to quickly navigate and/or to gain insight into the connectivity of desired pathway components. Additionally, the user might use back and forward buttons to facilitate the navigation. They can be found on the left and right upper corner of the navigation window. These buttons will also highlight a specific graph icon based on a mouse-over action, giving an additional insight to what destination would he/she arrive in case of clicking the Back- or the Forward-button, respectively. This will help to keep track of navigation processes and will avoid to search for the previous pathway in multiple open windows.

\subsubsection{Aggregated Links View}

The graph icons provide an overview of all pathways linked to the current focus pathway, but they cannot show the frequency a particular target pathway is being referenced from the nodes of the focus pathway. Accordingly, a statistical data visualization is desired. Instead of integrating it into the main window, we decided to use a multiple views approach that is a widely used technique to visualize different kind of data simultaneously. This method provides the possibility of choosing the most suitable visualization technique for a specific view and data set [7]. Guided by these ideas we added a separate interactive diagram placed at the right upper corner in a tab, called aggregated links view, to present these statistical data. Figure 6 shows a screenshot excerpt of this diagram. As it resembles the navigation glyphs used in the focus

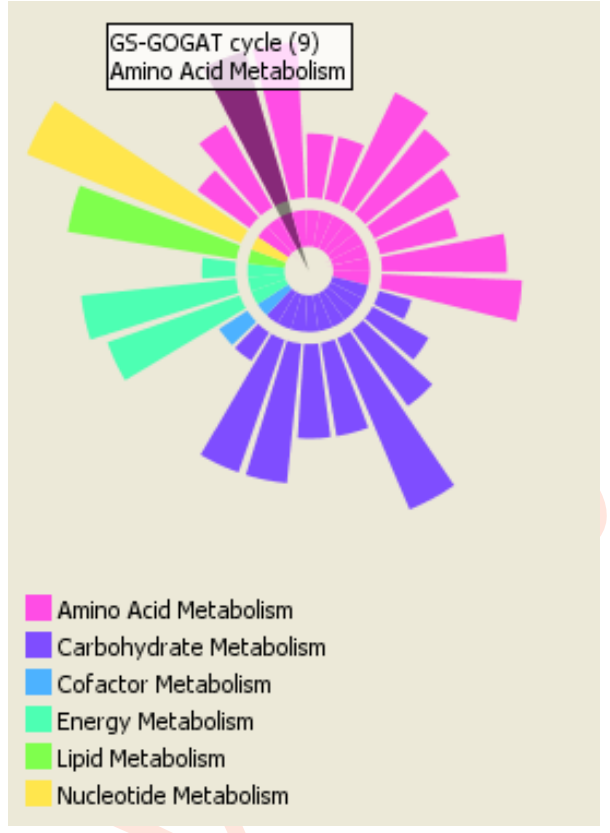

Fig. 6. Link rose diagram. The "GS-GOGAT cycle" is selected triggering a specific petal to be shadowed in a more saturated color. A mouse-over action shows the name of the pathway in the first line of a tooltip, while the number in brackets reveals the total number of links from the focus pathway to the selected pathway. The second line of the tooltip shows the name of the MetaCrop pathway group that the selected pathway belongs to. Users can instantly notice strongly and/or loosely connected pathways. The legend below helps in identification of different pathway groups. The color-coding of the groups is persistent for specific group, no matter where we navigate to.

pathway and is able to guide the user such as a traditional compass rose metaphor, we called it link rose. In contrast to the navigation glyphs that represent the links of a particular pathway node, it displays statistical data above all links to other connected pathways in context of the current focus pathway. Thus, it enables users to compare all outgoing pathway links and to show which pathways are strongly connected to the focus pathway.

Mouse-over actions on a rose petal will show a tooltip with the name of the corresponding pathway, followed by the number of nodes linked to that specific pathway. The tooltip also presents the pathway group name in the second text line. This gives an additional, textual dimension of insight on the connectivity of a specific graph to other pathways. Following the idea of several coordinated views [21], a click on a rose petal has also an effect on other parts of our visualization approach (besides to highlight the corresponding petal), namely that all graph nodes referencing to the selected pathway are also highlighted in the navigation window. This possibility enables users to immediately identify components connected to a particular pathway, and thus, enhancing the comprehension of interconnections of different graph components and providing a better overview on the entire network structure. Moreover, if we subsequently navigate to any target pathway, all shared nodes (clones) will be selected in the target pathway consequently revealing the common components between these pathways. A more detailed navigation and exploration example is given in Section 5 .

\subsection{Scalability}

Our visualization and interaction approach scales well when using the MetaCrop database consisted of 38 pathways. We are aware that the graph icons discussed in Section 4.2 depend on size and resolution of the screen as well as on the number of pathways links being visualized. Theoretically, if the display size and resolution decreases or the number of the pathways gets large enough, our graph icons would be minimized so much that it would be hard to recognize them. 


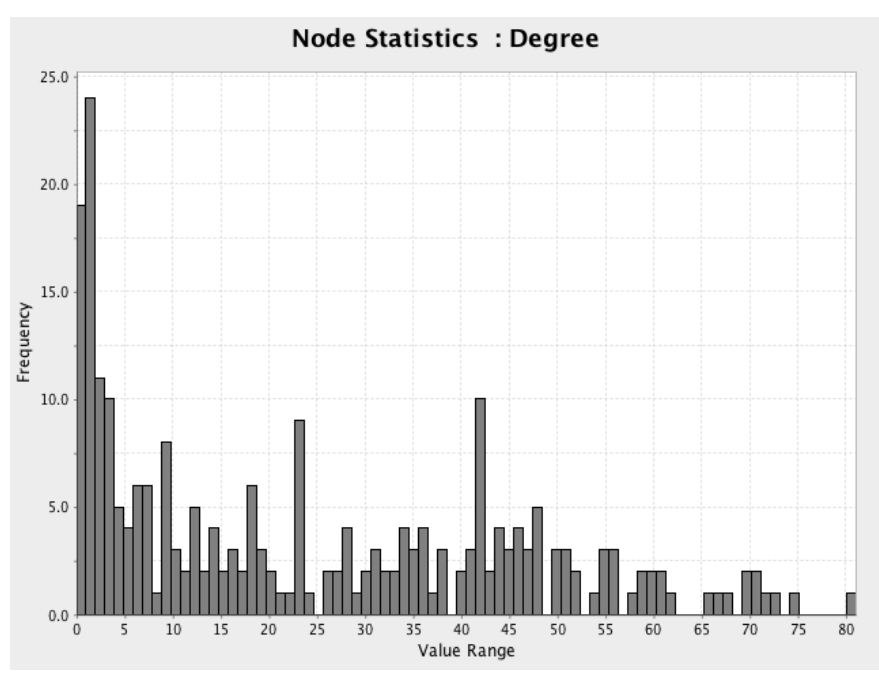

Fig. 7. The figure shows the number of links to other pathways (same substance in other pathways) for the reference pathways of KEGG ("Frequency" means number of pathways, e.g., there are 10 pathways which have connections to 42 pathways each). The largest number of links has the pathway Inositol phosphate metabolism, with substances of this pathway found in 81 other pathways.

Larger data sets, such as KEGG [10] that contains the largest collection of pathways and also both signaling and metabolic pathways, can demonstrate the scalability of our approach. Therefore, we analyzed KEGG focusing on substances (the most commonly shared elements between different pathways) and found that most of the pathways (about 95\%) have less than 62 links to other pathways in practical analysis sessions, as shown in the Figure 7. With these findings in mind, we can conclude that the application scales without any difficulties for KEGG in most cases: we used a laptop with a screen resolution of $1280 \times 800$ pixels and loaded a pathway with 81 links, which is the extreme case in Figure 7. We found out that the graph icons scale even in such extreme cases, although the individual nodes and edges in some of the graph icons were less distinguishable. Keeping in mind that nowadays standard office displays have even larger resolutions, we do not expect any scalability problems.

As mentioned before, we have also tested our approach with the KEGG data set. Currently, the mapping of KEGG hierarchies/groups is not completely implemented. There are two potential limitations: deeper hierarchies, i.e., more levels, and/or broader hierarchies, i.e., many different groups. The latter issue can easily be solved by using another color schema. The fact that each pathway group has a fixed position around the navigation window facilitates the perception additionally. This idea does not help with deeper levels. However, there are ways to overcome these constrains and we present them in the future work section of our paper.

\subsection{Implementation and Timing}

The VANTED system offers a comfortable way to implement various plugins as separate projects. Those can also be easily added by users later and allow a great deal of flexibility in the implementation process and possibilities for building various improvements as well as the embedding of new (visualization) techniques or tools. By utilizing this approach, the users of the VANTED system are able to customize the functionality according to their needs. Nevertheless, some interventions on VANTED's core libraries were necessary in order to implement all our desired features.

The time to download the data set depends on the Internet connection and the size/number of the desired pathways. Once all pathways are completely downloaded, users are able to load a focus pathway into the navigation window in 1-2 seconds in worst case. This includes the computation of the link connections to other pathways and the gener- ation of the aggregated links view. Additionally, it might take at most 2-3 seconds to generate the graph icons in case the focus pathway is strongly connected (40-50 links) to target pathways. Based on the figures in Figure 7, we can safely conclude that most of the interactions will occur in a real-time. Worst case situations occur within the unit task level [6]. We have tested our VANTED plugin using a Dell Latitude E6400 laptop with a $2.53 \mathrm{GHz}$ Intel Core 2 Duo processor and $3 \mathrm{~GB}$ of RAM running Windows XP.

\section{Use-Case Scenario}

In this section we will present the way our plugin is used. Usually, the users download the pathways they are interested in or the whole MetaCrop data set. In standard visualization approaches the navigation and exploration gets more complicated if the number of pathways being explored increases, and simultaneously the contextual information about the interconnectivity of different components is lost due to the reasons explained in previous sections of the paper.

After activating our plugin, users will be able to gain insight into the interconnectivity immediately. The graph icons arranged around the focus pathway are not merely links to other pathways as they provide an overview of the overall connectivity of the focus pathway (cf. Figure 4). Our aggregated links view provides more specific information about the frequency of the connections to each and specific pathways. These views together with various interaction and brushing techniques will guide the users to interactively explore the pathways. If a certain petal of the link rose is relatively long compared to the others, we might conclude that the corresponding pathway shares a considerable amount of components with our focus pathway currently displayed in the navigation window. We will explain the navigation process by means of Figure 8. Let us assume that our user have navigated to the Pentose phosphate pathway and noticed (cp. the upper part of the figure) that the link rose has a petal that is longer than the others. After a mouse-over event, he/she discovers that the name of the pathway is Calvin cycle and that there are 16 links to that particular pathway. If he/she clicks on the rose petal, all the nodes referencing that pathway will be selected. This strong interconnection between Carbohydrate Metabolism (where Pentose phosphate pathway belongs to) and Energy Metabolism (where Calvin cycle belongs to) can be further investigated by selecting the Calvin cycle.

After clicking the graph icon, the user navigates to the desired target pathway (see the bottom part of Figure 8). Previously selected nodes will remain selected if they exist in the new navigated pathway. We notice that several nodes in the Calvin cycle are now selected. This example demonstrates that the plugin makes it rather easy to identify the connectivity context of various pathways and facilitates the navigation between them.

Another investigation could start with the Sucrose breakdown pathway (monocots.). Again, the link rose has a petal that is much longer than the others. After a mouse-over event, the user discovers that the name of the pathway is Sucrose breakdown pathway (dicots.) and that there are 54 links to that particular pathway. Clicking on the rose petal selects all the nodes referencing that pathway. In this case, only three nodes are not selected, which clearly shows that this pathway is strongly interconnected with the selected one. After clicking the graph icon and navigating to the desired target pathway Sucrose breakdown pathway (dicots.) a biologist will immediately recognize, that we are navigating in our sample through the same metabolic pathway. In this case it shows the differences between an important pathway in monocotyledons (monocots) and dicotylodons (dicots).

\subsection{Feedback from Biologists}

We discussed our plugin in three user sessions with four domain experts from the IPK Gatersleben. These experts are biologists and biochemists used to work with metabolic pathways and are familiar with pathway databases such as KEGG and MetaCrop, as well as with the VANTED system. After a short introduction in the usage of the plugin and the idea of the navigation method (introduction was less then five minutes), we ask the experts to work with the plugin to investigate the 

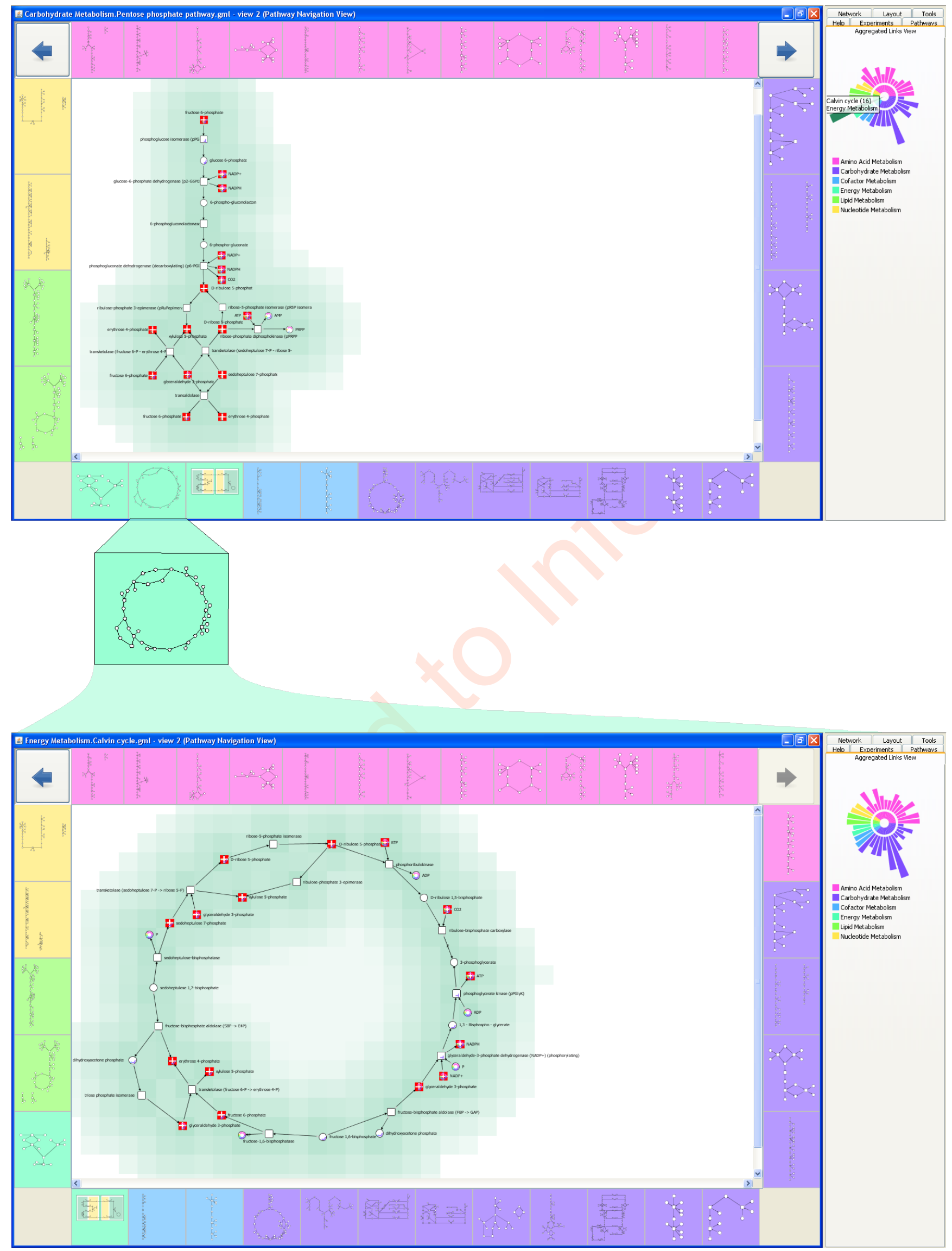

Fig. 8. Navigation between two pathways. The selection of the nodes is persistent even when navigating in the new pathway. A feature that helps to discover shared components. 
metabolic pathways stored in the MetaCrop system and report positive and negative aspects.

In general the feedback was very positive. Users described the navigation through pathways as intuitive and very helpful, even as "cool". The used colors and the size of the glyphs were considered appropriate, the speed of navigation (e.g., replacement of one pathway by the next one) mostly as fast enough. Especially the interaction between the link rose and the pathway was considered useful for selecting nodes occurring in another pathway.

However, there were also several suggestions by the domain experts. Although most liked the concept of presenting only those pathways as graph icons which are connected to the current pathway of interest, one user would prefer to have all other pathways (also currently not connected ones) as graph icons placed around the current pathway. Users familiar with the MetaCrop pathways had no problem to navigate to specific pathways (graph icons), but some recommended nevertheless to include the name of the pathway in the icon (currently the name is only shown after moving the mouse over the icon). Finally, one user recommended to optionally restrict linkage of substances and avoid co-substances (e.g., ATP, $\mathrm{H}_{2} \mathrm{O}$ ). This would considerably reduce the number of interconnected pathways.

The users found the method very helpful to navigate through pathways but recommended additional functionality to make it more useful for their work such as the ability to map measured data onto nodes or to see changes in fluxes over reactions. It should be noted that these are aspects which are supported by the VANTED system (e.g., fluxes can be represented by different edge width and additional data such as metabolomics data by additional coloring of nodes or including of diagrams into nodes) and are already used by some of the experts for their data analysis. Such functionality is already possible with our plugin, however, as it is not focus of this study, we did not extend the discussion with the users into this direction.

\section{CONCLUSion AND FUtURE WORK}

We presented in this paper a solution to guide the navigation and exploration process of interconnected pathway visualization and discussed it in context of biochemical networks. Our visualization approach supports the analyst to get an overview to connected pathways if he/she is working within the currently focused pathway. This problem appears simple, but it still not sufficiently solved in existing visualization tools.

Our main contribution is to extend a complex system used in practice with novel and intuitive visualization and interaction techniques to guide the domain expert during the exploration and navigation process. Our tool uses well-known techniques, such as glyphs [29,30], brushing [25], and topological information of the target pathways (realized by the graph icons), to provide the analyst with more meaningful context information in hierarchically structured biochemical pathways.

As future work, we plan to improve different issues regarding the visualization and interaction possibilities as well as the availability of our VANTED plugin. They are listed in the following:

- We address a particular and urgent concern regarding the use of the VANTED system by biologists and bioinformaticians. Hence, our plan is to publish our plugin on SourceForge [24], where users can easily download it, and programmers can use our source code for further improvements or for adding additional features.

- Recent research results in large graph exploration, such as the DOI-approach of van Ham and Perer [28], could be interesting for our system too. We plan to analyze the possibility of integrating such or similar techniques.

- A limitation mentioned before is to regard the visualization of data sets with a deeper level of hierarchies. Currently, we can only support two-level hierarchies. However, this is enough for the discussed data sets. Theoretically, our approach could be extended by using the sunburst approach presented by Stasko and Zhang [26] or Richard et al. [20].
- The link rose diagram could be extended by additional statistical data. The color-coding of the rose diagram and the navigation glyphs should be improved as well.

- Our small usability study with biologists helped us to identify some potential improvements. One suggestion aimed to show all pathways as graph icons including pathways that are currently not connected. We will analyze this proposal, but it would probably lead to a overloaded user interface. Another improvement is to embed the name of a pathway into its graph icon as well as to provide some filtering techniques to reduce the number of interconnected pathways.

- VANTED is a powerful tool used by many domain experts. Thus, we are convinced that our plugin can facilitate their work. Nevertheless, a carefully designed larger user study or evaluation might detect more concrete or conceptual problems of our approach, which can be improved in a successive stage. A comparative study with MetaVis [4] is desirable as well.

\section{REFERENCES}

[1] M. Albrecht, A. Kerren, K. Klein, O. Kohlbacher, P. Mutzel, W. Paul, F. Schreiber, and M. Wybrow. A graph-drawing perspective to some open problems in molecular biology. Technical report, Lehrstuhl XI für Algorithm Engineering, Fakultät für Informatik, Technische Universität Dortmund, June 2008.

[2] M. Albrecht, A. Kerren, K. Klein, O. Kohlbacher, P. Mutzel, W. Paul, F. Schreiber, and M. Wybrow. On open problems in biological network visualization. In Proc. International Symposium on Graph Drawing (GD '09), volume 5849 of $L N C S$, pages 256-267. Springer, 2010.

[3] C. Bachmaier, F. J. Brandenburg, M. Forster, P. Holleis, and M. Raitner Gravisto: Graph visualization toolkit. In J. Pach, editor, Proc. International Symposium on Graph Drawing (GD'04), volume 3383 of LNCS, pages 502-503. Springer, 2005.

[4] R. Bourqui, V. Lacroix, L. Cottret, D. Auber, P. Mary, M.-F. Sagot, and F. Jourdan. Metabolic network visualization eliminating node redundance and preserving metabolic pathways. BMC Systems Biology, 1:29, 2007.

[5] U. Brandes, T. Dwyer, and F. Schreiber. Visual understanding of metabolic pathways across organisms using layout in two and a half dimensions. Journal of Integrative Bioinformatics, 1(1):119-132, 2004.

[6] S. Card, J. Mackinlay, and B. Shneiderman, editors. Readings in Information Visualization: Using Vision to Think. Morgan Kaufmann, 1999.

[7] C. Görg, M. Pohl, E. Qeli, and K. Xu. Visual Representations. In Kerren et al. [12], pages 163-230.

[8] E. Grafahrend-Belau, S. Weise, D. Koschützki, U. Scholz, B. H. Junker, and F. Schreiber. MetaCrop - a detailed database of crop plant metabolism. Nucleic Acids Research, 36:D954-D958, 2008.

[9] B. H. Junker, C. Klukas, and F. Schreiber. VANTED: A system for advanced data analysis and visualization in the context of biological networks. BMC Bioinformatics, 7:109, 2006.

[10] M. Kanehisa, S. Goto, S. Kawashima, and A. Nakaya. The KEGG databases at GenomeNet. Nucleic Acids Research, 30(1):42-46, 2002.

[11] A. Kerren. Interactive visualization and automatic analysis of metabolic networks - a project idea. Technical report, Institute of Computer Graphics and Algorithms, Vienna University of Technology, Austria, 2003.

[12] A. Kerren, A. Ebert, and J. Meyer, editors. Human-Centered Visualization Environments. LNCS Tutorial 4417. Springer, 2007.

[13] C. Klukas and F. Schreiber. Dynamic exploration and editing of KEGG pathway diagrams. Bioinformatics, 23(3):344-350, 2007.

[14] C. Klukas and F. Schreiber. Integration of -omics data and networks for biomedical research. Journal of Integrative Bioinformatics, 7:112, 2010.

[15] R. S. Laramee and R. Kosara. Challenges and Unsolved Problems. In Kerren et al. [12], pages 231-254.

[16] M. Lungu and K. Xu. Biomedical Information Visualization. In Kerren et al. [12], pages 311-342.

[17] K. Misue, P. Eades, W. Lai, and K. Sugiyama. Layout adjustment and the mental map. Journal of Visual Languages and Computing, 6:183-210, 1995.

[18] F. Nightingale. Notes on Matters Affecting the Health, Efficiency, and Hospital Administration of the British Army. Harrison \& Sons., 1858.

[19] R. Rao and S. K. Card. The table lens: merging graphical and symbolic representations in an interactive focus+context visualization for tabular 
information. In CHI '94: Conference companion on Human factors in computing systems, page 222. ACM, 1994.

[20] J. S. Richard, R. Catrambone, M. Guzdial, and K. Mcdonald. An evaluaion of space-filling information visualizations for depicting hierarchical structures. International Journal of Human-Computer Studies, 53:663694, 2000.

[21] J. C. Roberts. Exploratory visualization with multiple linked views. In A. MacEachren, M.-J. Krak, and J. Dykes, editors, Exploring Geovisualization. Elseviers, December 2004.

[22] M. Rohrschneider, C. Heine, A. Reichenbach, A. Herren, and G. Schemermann. A novel grid-based visualization approach for metabolic networks with advanced focus\&context view. In Proc. International Symposium on Graph Drawing (GD '09), volume 5849 of LNCS, pages 268-279. Springer, 2010.

[23] P. Saraiya, C. North, and K. Ducal. Visualizing biological pathways: requirements analysis, systems evaluation and research agenda. Informaion Visualization, 4(3):191-205, 2005.

[24] SourceForge. Find and develop open source software, 2009. http://sourceforge.net/.

[25] R. Spence. Information Visualization: Design for Interaction. Prentice Hall, 2nd edition, 2007.

[26] J. Stasko and E. Chang. Focus+context display and navigation techniques for enhancing radial, space-filling hierarchy visualizations. In INFOVIS 'O0: Proc. IEEE Symposium on Information Vizualization 2000, page 57, Washington, DC, USA, 2000. IEEE Computer Society.

[27] M. Streit, M. Kalkusch, K. Kashofer, and D. Schmalstieg. Navigation and exploration of interconnected pathways. Compute. Graph. Forum, 27(3):951-958, 2008.

[28] F. van Ham and A. Perer. Search, show context, expand on demand: Supporting large graph exploration with degree-of-interest. IEEE Transactions on Visualization and Computer Graphics, 15:953-960, 2009.

[29] M. O. Ward. Xmdvtool: integrating multiple methods for visualizing multivariate data. In VIS '94: Proc. Conference on Visualization '94, pages 326-333, Los Alamitos, CA, USA, 1994. IEEE Computer Society Press.

[30] M. O. Ward. A taxonomy of glyph placement strategies for multidimensional data visualization. Information Visualization, 1:194-210, 2002.

[31] C. Ware. Information Visualization: Perception for Design. Morgan Kaufmann, Ind edition, 2004.

[32] S. Wise, I. Grosse, C. Klukas, D. Koschützki, U. Scholz, F. Schreiber, and B. H. Junker. Meta-All: a system for managing metabolic pathway information. BMC Bioinformatics, 7:465, 2006. 